\title{
Maximising the Sensitivity and Specificity of Non- Contact Tonometry in Glaucoma Screening
}

\author{
STEPHEN A. VERNON, ${ }^{1}$ SUSAN J. JONES, ${ }^{2}$ DONALD J. HENRY ${ }^{2}$ \\ Nottingham
}

\begin{abstract}
Summary
Data from a glaucoma screening study involving $88.5 \%$ of the population age 50 and over of a single handed general practitioner were reanalysed to determine the effect of altering the protocol for intraocular pressure assessment and the effect of changing the referral threshold.

The predictive power of the Keeler Pulsair noncontact tonometer was found to decrease from $22.5 \%$ at four pulses per eye to $12.3 \%$ when only one pulse per eye was used, with a reduction of sensitivity from $91.7 \%$ to $75 \%$.

The sensitivity of the same device fell from $91.7 \%$ if all patients with an IOP $>21 \mathrm{mmHg}$ were deemed as having a positive screen, to $41.6 \%$ when only patients with an IOP $>26 \mathrm{mmHg}$ were considered for referral.

To create a balance between high sensitivity and acceptable predictive power of a positive result in a population where $50 \%$ of glaucoma sufferers are known prior to screening, we advise that four pulses per eye should be used with an IOP of $>22 \mathbf{m m H g}$ used as the significant finding indicating that the patient required referral.
\end{abstract}

Tonometry alone has a poor reputation as a screening tool in the detection of glaucoma, as witnessed by a number of epidemiological studies. ${ }^{1,2,3}$ These studies used a definition of glaucoma which included a demonstration of field loss, although only one of the studies performed visual field analysis in all of the population studied and this study was limited to those individuals aged between 55 and 69 years. $^{3}$

Visual field loss is now known to be a relatively late feature of glaucomatous damage to the optic nerve ${ }^{4}$ and treatment is often advisable in the absence of demonstrable field loss if other risk factors are present. ${ }^{5,6.7}$
In Great Britain, many optometrists screen for glaucoma but a recent study has found that, in one major city, $50 \%$ of optometrists will not refer a patient for ophthalmological assessment unless the IOP is $>24 \mathrm{mmHg}$. Seventy-one per cent of the optometrists used non-contact tonometers (NCT) but $35 \%$ of these were measuring the IOP using only one or two pulses per eye.

We have previously reported the results of a pilot study on the use of semi-automated equipment to screen for glaucoma in the community utilising non-ophthalmically trained staff. ${ }^{10}$ This study, examining $88.5 \%$ of a population aged 50 and over, used the fol-

From: ${ }^{1}$ Academic Unit of Ophthalmology, University of Nottingham. ${ }^{2}$ Department of General Practice, University of Nottingham.

Correspondence to: Mr S. A. Vernon FRCS, FCOphth., The Academic Unit of Ophthalmology, University Hospital, Nottingham. 
lowing criteria for defining a positive, i.e. a patient requiring treatment for IOP related disease.

(1) IOP $>22 \mathrm{mmHg}+$ pathologically cupped disc with field loss-or

(2) IOP $>22 \mathrm{mmHg}$ on two hospital visits in association with two or more of six pathological disc parameters on three dimensional disc assessment despite normal fields-or

(3) IOP $>30 \mathrm{mmHg}$ on two hospital visits.

All subjects in the study had tonometry using a Keeler 'Pulsair' NCT taking the mean of four pulses per eye, visual field analysis using a Henson CFS2000 field screener and 'blind' ophthalmoscopic examination of the optic discs by an experienced ophthalmologist. The sensitivity and specificity of the NCT was found to be $91.7 \%$ and $95.6 \%$ respectively.

This paper examines the effect of altering the referral criteria in a tonometry based screening programme and the effect of reducing the number of pulses per eye to determine the IOP.

\section{Materials and Methods}

Data from the aforementioned study ${ }^{9,10}$ was reanalysed to calculate the sensitivity, specificity and predictive power of a positive screen $(\mathrm{PP}+\mathrm{ve})$ when the Pulsair was used to screen a population aged 50 years and over for glaucoma. Full details of the original protocol are described elsewhere. ${ }^{9}$ In the first instance, we examined the effect of varying the referral IOP whilst maintaining the Pulsair protocol as previously described i.e. the mean of four pulses per eye.

We also analysed the effect of reducing the number of pulses per eye that were used to

Table I The effect of varying the IOP referral threshold

\begin{tabular}{lccc}
\hline $\begin{array}{l}\text { Referral } \\
\text { Threshold } \\
(\mathrm{mmHg})\end{array}$ & $\begin{array}{c}\text { Sensitivity } \\
(\%)\end{array}$ & $\begin{array}{c}\text { Specificity } \\
(\%)\end{array}$ & PP+ve (\%) \\
\hline$>21$ & 91.7 & 92.5 & 14.5 \\
$>22$ & 91.7 & 95.6 & 22.5 \\
$>23$ & 83.3 & 97.5 & 32.3 \\
$>24$ & 75.0 & 98.5 & 41.0 \\
$>25$ & 58.3 & 99.3 & 54.0 \\
$>26$ & 41.6 & 99.7 & 62.5 \\
\hline
\end{tabular}

determine the IOP at screening whilst maintaining on IOP of $>22 \mathrm{mmHg}$ as an indication for referral. The effect of three pulses per eye was obtained by omitting the fourth pulse reading from the calculation of the mean. To obtain the effect of two pulses per eye we omitted the last two readings and for a single pulse the last three readings.

\section{Results}

Twelve individuals were found to require treatment for raised IOP in the original study and serve as true positives for the purposes of this study.

Table I indicates the variation in sensitivity, specificity and $\mathrm{PP}+$ ve found to occur at different IOP referral thresholds.

Table II indicates the variation in sensitivity, specificity and PP+ve found to occur when the number of NCT pulses per eye is gradually reduced from four to one.

\section{Discussion}

Previous epidemiological studies from western countries have indicated that between 33 and $50 \%$ of glaucoma sufferers in the population are known to the medical profession prior to screening. ${ }^{1,2,3}$ Our study, in which great care was taken to reduce false negatives to a minimum, found a similar result despite the increasing popularity of optometrist screening. ${ }^{9}$ In a population with no known cases of glaucoma where the prevalence of the disease is $2 \%$, a test with a $90 \%$ sensitivity and $95 \%$ specificity will have a $\mathrm{PP}+\mathrm{v}$ of $27 \%$. $^{11}$

In this study when the IOP referral level is raised, a high $\mathrm{PP}+\mathrm{ve}$ is achieved at the expense of poor sensitivity.

This decreases the value of the screening programme to the community although it improves the cost per case detected. Clearly an acceptable balance is required.

In our original study, four pulses per eye

Table II The effect of reducing the number of pulses per eye

\begin{tabular}{lccc}
\hline $\begin{array}{l}\text { No of } \\
\text { Pulses/eye }\end{array}$ & $\begin{array}{c}\text { Sensitivity } \\
(\%)\end{array}$ & $\begin{array}{c}\text { Specificity } \\
(\%)\end{array}$ & PP+ve (\%) \\
\hline 4 & 91.7 & 95.6 & 22.5 \\
3 & 83.3 & 95.5 & 20.4 \\
2 & 83.3 & 94.1 & 16.7 \\
1 & 75.0 & 92.5 & 12.3 \\
\hline
\end{tabular}


were obtained from each patient in an average of two minutes, including an explanation of the test. This study has shown that reducing the number of pulses per eye to two, reduces the PP+ve by $25 \%$.

The sensitivity of optometrists when screening for glaucoma in the UK is unknown as details of false negatives are unavailable. This study indicates that screeners who raise the referral IOP in addition to using fewer than four pulses per eye significantly reduce the sensitivity and potential $\mathrm{PP}+\mathrm{ve}$ of the test.

Our studies with NCT in a population at risk from glaucoma in whom half the cases have been detected previously, indicate that to achieve a balance between high sensitivity $(>90 \%)$ and $\mathrm{PP}+\mathrm{ve}(>20 \%)$, at least four pulses per eye should be used and the threshold for referral (with the Pulsair NCT) should remain $\mathrm{IOP}>22 \mathrm{mmHg}$.

\section{References}

${ }^{1}$ Hollows FC and Graham PA: Intraocular pressure, glaucoma and glaucoma suspects in a defined population. Br J Ophthalmol 1966, 58: 570-86.

${ }^{2}$ Leske MC, Podgor M, Ederer F: An evaluation of glaucoma screening methods. Invest Ophthalmol Vis Sci 1982, 22 (suppl) 128.

${ }^{3}$ Bengtsonn B: The prevalence of glaucoma. $\mathrm{Br} \mathrm{J}$ Ophthalmol 1981, 65: 46-9.

${ }^{4}$ Quigley HA, Addicks EH, Green WR: Optic nerve damage in human glaucoma. Arch Ophthalmol 1982, 100: 135-46.

${ }^{5}$ Smith RJH: Ocular hypertension Res Clin Forum 1980, 2: 129-31.

${ }^{6}$ Mills KB: Ocular hypertension Res Clin Forum 1985, 7: 81-4.

${ }^{7}$ Goldmann $\mathrm{H}$ : An analysis of some concepts concerning chronic simple glaucoma. Am J Ophthalmol 1975, 80: 409-13.

${ }^{8}$ Vernon SA and Henry DJ: Do optometrists screen for glaucoma? Eye 1989, 3: 743-6.

${ }^{9}$ Vernon SA, Henry DJ, Cater L, Jones SJ: Screening for glaucoma in the community by non-ophthalmologically trained staff using semi-automated equipment. Eye 1990, 4: 88-97.

${ }^{10}$ Jones SJ, Vernon SA, Cater L, Henry DJ: Costing a community based screening programme for the detection of glaucoma. Eye 1990, 4: 98-102.

${ }^{11}$ Leske MC and Hawkins BS: Screening: relationship to diagnosis and therapy. In Duanne TD, Jaeger EA eds. Clinical Ophthalmology. Philadelphia, Philadelphia, Harper and Row, 1987, 3 (54): 1-16. 